\title{
CHALLENGES FOR PEDIATRIC HEMATOLOGY ONCOLOGY IN PAKISTAN DURING CORONAVIRUS PANDEMIC
}

\author{
Rahat Ul Ain ${ }^{1}$ and Mahwish Faizan ${ }^{1}$ \\ ${ }^{1}$ The Children's Hospital and the Institute of Child Health
}

June 28, 2020

\begin{abstract}
Background: During COVID-19 pandemic, as the prime care is directed towards the management of infected cases, patients with non-communicable diseases are subject to lack of attention. Pediatric Hematology Oncology patients demand holistic care therefore might get affected tremendously during this pandemic. Procedure: An online cross-sectional survey was carried out among the Pediatric Hematologists-Oncologists from all over Pakistan in order to assess the pandemic effects. Responses were received online and data analyzed in SPSS 16.0. Results: Survey was sent to 96 Pediatric Hematologists-Oncologists from 14 different centers with $50 \%$ response rate. Majority (62\%) were working in public sector hospitals, $14 \%$ reported to have stopped registering new patients, $68 \%$ reported reduced overall number of patients and $64 \%$ reported reduction of patients for scheduled chemotherapies; $78 \%$ reported affected supportive care services while $70 \%$ reported closure of various units of the department. Affected supplies, specialized investigations, tumor boards, oncological surgeries, radiation therapies, stem cell transplant and academic activities were also reported. Availability of PPE was satisfactory, $62 \%$ reported unavailability of isolated febrile neutropenia rooms, $58 \%$ reported inability to exercise social distancing in ward while $90 \%$ reported to exercise short staff rosters. Significant level of personal and family health concern $(\mathrm{M}>2.5)$ and stress $(>3.0)$ during the pandemic were reported by pediatric hematologists/oncologists. Conclusion: Apart from overwhelming issues of COVID-19 patients and their doctors, patients with non-communicable diseases and their treating physicians are also tremendously affected during the pandemic and interrupted management of pediatric hematology oncology patients during pandemic may lead to devastating long term effects.
\end{abstract}

\section{INTRODUCTION:}

During the COVID-19 pandemic, the prime care is directed towards the management of huge burden of infected cases therefore, a large number of patients with non-communicable diseases are subject to lack of attention and interrupted routine care. Pediatric Hematology Oncology patients demand holistic care therefore these patients might get affected tremendously during this pandemic.

On 31 December 2019, the first case of pneumonia of unknown cause was reported in Wuhan, China. The outbreak was declared as a Public Health Emergency of International Concern on 30 January 2020. The World Health Organization (WHO) announced a name for the new coronavirus disease: COVID-19 on 11 February $2020^{1}$. This novel corona virus disease spread rapidly worldwide, with epidemics in around 215 countries and meeting the definitions of a pandemic ${ }^{2}$. Estimated case fatality rates are around $2 \%$ which may rise to $15 \%$ in aged patients ${ }^{3}$. As there is no vaccine or specific antiviral therapy yet available therefore, only prevention or delay community spread of the virus can be done by containment and laborious case finding. In many countries where COVID-19 became widespread, quarantine and social distancing measures have been adopted to slow its further spread ${ }^{4}$.

In Pakistan, first confirmed case of COVID-19 was reported on $26^{\text {th }}$ February $2020^{5}$. Country wide lock down was announced in the last week of March in order to control the increasing number of cases ${ }^{6}$. Patients 
with non-communicable diseases especially cancers are particular at risk and may be affected during this COVID-19 pandemic largely in two ways: elevated risk of severe COVID-19 disease, and disrupted delivery of cancer therapies due to quarantines, social distancing measures, and interrupted healthcare delivery.

The subspecialty of Pediatric Hematology/Oncology (PHO) has recently developed over few decades in Pakistan with around 15 specialized centers all over the country. Being a low-middle income country, major challenges in the management of these patients has been poor access and delayed presentation to the specialized centers, insufficient healthcare facilities, and lack of public awareness, illiteracy, poverty and malnutrition. On the canvas of these innate challenges and suboptimal care delivery to the pediatric hematology-oncology patients in a developing country, the emergence of COVID-19 pandemic has added up to the existing challenges. The objectives of this study were to determine the effects of COVID-19 pandemic over the patients of non-communicable diseases like pediatric hematology/oncology and the analyze the challenges faced by the Pediatric Hematologists/ Oncologist in a developing country during the pandemic.

\section{MATERIALS AND METHODS:}

An online cross-sectional survey was carried out among the Pediatric Hematologists-Oncologists working at different specialized centers from all over the country. The survey was opened for collection of responses on April $19^{\text {th }} 2020$ and closed on April $27^{\text {th }} 2020$. Responses were received online and the data was entered and analyzed in SPSS 16.0. The survey questionnaire comprised of 20 multiple choice questions pertaining to Pediatric Hematology-Oncology clinical practices, delivery of care and provision of services at various PHO centers in Pakistan along with the assessment of stress and health concerns among the treating Hematologists/Oncologists during the COVID-19 pandemic.

\section{RESULTS:}

The survey was sent to 96 Pediatric Hematologists-Oncologists across the country while only 53 responded with a response rate of $50 \%$. Out of the 53 responses, 50 responses were considered valid as per inclusion criteria of responses from Pediatricians or Pediatric Hematologist/Oncologists actively working in Pediatric Hematology/Oncology in Pakistan. The respondents belonged to 14 different centers from all over Pakistan: 9 centers from the province of Punjab, 4 from Sindh province (Karachi) and 1 center from Khyber-Pakhtunkhua province (Peshawar). Currently there is no Pediatric Hematology-Oncology center in the rest of two provinces of Pakistan (Baluchistan and Gilgit-Baltistan).

Majority of the respondents $(62 \%)$ were working in public sector hospitals with availability of Pediatric Oncology, Pediatric Hematology and/or Pediatric Bone Marrow Transplant facilities. Eighty two percent Pediatric Hematologist/Oncologists were working in the hospitals where COVID19 confirmed cases from general population were being catered. When asked about the registration of new patients of PHO during pandemic, $14 \%(\mathrm{n}=7)$ reported to have stopped registering new patients and $16 \%(\mathrm{n}=8)$ reported registering only selective patients. Reduced work load during the pandemic was reported by $68 \%$ respondents, reduction in number of patients coming for routine chemotherapies was reported by $64 \%$, affected supportive care services for PHO patients were reported by $78 \%$ while closure of various units of the PHO department (Outpatient department, post-treatment follow up clinics, outpatient transfusions/factor infusion clinics and high dependency unit) was reported by $70 \%$ respondents.

A substantial number of pediatric hematologist/oncologists reported having affected supplies of items that are commonly used in PHO units including infection prevention and control supplies, chemotherapeutic drugs, antibiotics, supportive care medicines and blood products (Table 1). The pandemic seriously affected important investigations needed to diagnose and manage major hematological/oncological disorders, multidisciplinary tumor boards, oncological surgeries, radiation therapies, stem cell transplantation and teaching and training activities of the post-graduate residents. Half of the clinicians (50\%) reported to have an institutional protocol available for the management of immunocompromised COVID19 patients and mentioned about the screening protocols and availability of COVID19 testing in pediatric hematology/oncology patients. None of the participants reported any confirm case of COVID19 among hematology/oncology patients or among their staff members till date (April $27^{\text {th }}$ 2020). Satisfactory availability of PPE was reported. Regard- 
ing ability to apply recommended measures for the prevention or management of COVID-19 in the pediatric oncology population ${ }^{7}, 62 \%$ respondents reported unavailability of isolated febrile neutropenia rooms, $58 \%$ reported to be unable to exercise social distancing in ward but $90 \%$ reported to be able to exercise short staff rosters with extended holiday period during the pandemic.

In order to assess the level of personal and family health concerns among the Pediatric Hematologists/Oncologists working during the pandemic, a 1-4 Likert scale was used $(1=$ Not concerned at all, $2=$ Slightly concerned, $3=$ Moderately concerned, and $4=$ Deeply concerned). Using the single sample t-test, the mean level of concern was calculated and evaluated whether the mean score was significantly above or below the mean (2.5). Significant levels of concern $(\mathrm{M}>2.5)$ were reported by all of the pediatric hematologists/oncologists regarding their personal health $[\mathrm{M}=3.26, \mathrm{SD}=0.78,95 \% \mathrm{CI}=(3.04,3.48)]$ and family health $[\mathrm{M}=3.64, \mathrm{SD}=0.60,95 \% \mathrm{CI}=3.47,3.81)]$. Similarly for the assessment of extent of stress among them, a 1-5 Likert scale was used ( $1=$ Not stressed at all, $2=$ To a small extent, $3=$ Somewhat stressed, $4=$ To a large extent, and $5=$ Completely stressed out) and significant level of stress $(>3.0)$ was also reported $[\mathrm{M}=3.00$, $\mathrm{SD}=0.73,95 \% \mathrm{CI}=(2.79,3.20)]$ by all of the participants.

\section{DISCUSSION:}

The COVID-19 pandemic has influenced health, economy and social practices worldwide. In Pakistan with a population of around 220 million, estimated new cases of cancer per year are between 170,000 and 200,000 . The developing countries endure the major burden of pediatric malignancies globally. Despite notable improvement in overall survival of pediatric cancers in high income countries, children with malignancies in developing countries are still battling with poor survival rates ${ }^{9}$. The COVID-19 pandemic has caused overloading of healthcare systems in all of the affected countries which has resulted in disruption of routine treatment of non-communicable diseases especially patients of hematology/oncology in whom an interruption in treatment may negatively affect the outcome ${ }^{10}$.

We can infer that an ample number of pediatric hematology oncology patients could not show up for their scheduled chemotherapies or interventions and the ones who could manage to present to the hospital have not been provided with the optimal routine management facilities. This breach in treatment and provision of suboptimal care may lead to devastating long term effects in the form of poor response to treatment or disease relapse. Apart from disruptions in patients' care and management, the pediatric hematologists/oncologists are also subject to academic, psychological and social problems. Increased challenges in the management of patients, limited personal protective equipment, and augmented concern of self-infection with fear of infecting their families have led to significantly increased stress among the Pediatric Hematologist/Oncologists across the country.

\section{CONCLUSIONS:}

The COVID-19 Pandemic has enormously affected the world. Apart from the overwhelming issues of COVID19 patients and the doctors treating these infected patients, the patients with non-communicable diseases and their specialist physicians who are not directly involved in the management of COVID-19 patients are also tremendously affected during the pandemic. Interrupted and suboptimal care of pediatric hematology oncology patients during pandemic may lead to devastating long term effects.

\section{CONFLICT OF INTEREST STATEMENT:}

There are no conflicts of interest to disclose.

\section{REFERENCES:}

1. The World Health Organization (WHO). Available at https://www.who.int/emergencies/diseases/novelcoronavirus-2019/events-as-they-happen Accessed May 2nd 2020.

2. The World Health Organization (WHO). Available at https://www.who.int/emergencies/diseases/novelcoronavirus-2019 Accessed May $2^{\text {nd }} 2020$. 
3. Wu Z, McGoogan JM. Characteristics of and Important Lessons From the Coronavirus Disease 2019 (COVID-19) Outbreak in China: Summary of a Report of 72314 Cases From the Chinese Center for Disease Control and Prevention. JAMA. 2020.

4. Wilder-Smith A, Freedman DO. Isolation, quarantine, social distancing and community containment: pivotal role for old-style public health measures in the novel coronavirus (2019-nCoV) outbreak. J Travel Med. 2020;taaa020.

5. "Two coronavirus cases confirmed in Pakistan - Pakistan Today". www.pakistantoday.com.pk. Retrieved 4 March 2020.

6. "Sindh decides to go into COVID-19 lock down". The Express Tribune. 21 March 2020. Retrieved 22 March 2020.

7. Bouffet E, Challinor J, Sullivan M, et al. Early advice on managing children with cancer during the COVID-19 pandemic and a call for sharing experiences. Pediatr Blood Cancer. 2020; e28327

8. International Agency for Research on Cancer Globocan. Pakistan fact sheet. 2018. [ https://gco.iarc.fr/today/data/factsheets/populations/586-pakistan-fact-sheets.pdf]

9. Usmani NG. Pediatric Oncology in the Third World. Curr Opin Pediatr. 2001; 13(1):1-9

10. Saini KS, Heras B, Castro J, et al. Effect of the COVID-19 pandemic on cancer treatment and research. Lancet Haematol. 2020.

\section{Hosted file}

table PHO COVID19.docx available at https://authorea.com/users/337264/articles/462886challenges-for-pediatric-hematology-oncology-in-pakistan-during-coronavirus-pandemic 